\title{
Tracing Electron-Ion Recombination in Nanoplasmas Produced by Extreme-Ultraviolet Irradiation of Rare-Gas Clusters
}

\author{
B. Schütte, ${ }^{1, *}$ F. Campi, ${ }^{2}$ M. Arbeiter, ${ }^{3}$ Th. Fennel, ${ }^{3}$ M. J. J. Vrakking, ${ }^{1}$ and A. Rouzée ${ }^{1}$ \\ ${ }^{1}$ Max-Born-Institut, Max-Born-Strasse 2 A, 12489 Berlin, Germany \\ ${ }^{2}$ Department of Physics, Lund University, P.O. Box 118, SE-221 00 Lund, Sweden \\ ${ }^{3}$ Institute of Physics, University of Rostock, Universitätsplatz 3, 18051 Rostock, Germany
}

(Received 27 January 2014; published 25 June 2014)

\begin{abstract}
We investigate electron-ion recombination in nanoplasmas produced by the ionization of rare-gas clusters with intense femtosecond extreme-ultraviolet (XUV) pulses. The relaxation dynamics following XUV irradiation is studied using time-delayed 790-nm pulses, revealing the generation of a large number of excited atoms resulting from electron-ion recombination. In medium-sized Ar-Xe clusters, these atoms are preferentially created in the Xe core within 10 ps after the cluster ionization. The ionization of excited atoms serves as a sensitive probe for monitoring the cluster expansion dynamics up to the ns time scale.

DOI: 10.1103/PhysRevLett.112.253401

PACS numbers: 36.40.Gk, 32.80.Fb, 33.60.+q, 52.50.Jm
\end{abstract}

In the last few years, pioneering experiments using newly developed extreme-ultraviolet (XUV) and $\mathrm{x}$-ray free-electron lasers have allowed the investigation of the ionization and dissociation of rare-gas clusters under intense short-wavelength radiation. High charge states were observed in Xe clusters reaching up to $\mathrm{Xe}^{8+}$ at an intensity of $2 \times 10^{13} \mathrm{~W} / \mathrm{cm}^{2}$ and a wavelength of $98 \mathrm{~nm} \mathrm{[1]}$. Moreover, ionization stages beyond $\mathrm{Xe}^{26+}$ were found at a wavelength around $1.5 \mathrm{~nm}$ and for pulse intensities exceeding $10^{16} \mathrm{~W} / \mathrm{cm}^{2}[2,3]$. While these results are impressive, the interpretation and physical understanding of the mechanisms involved has, up until now, been far from complete. A promising route towards gaining additional insight into the dynamical mechanisms are timeresolved experiments.

Electron-ion recombination processes have been predicted to play a crucial role in the cluster expansion process resulting from the interaction with an intense XUV or x-ray laser pulse [4-8], where the number of ions with higher charge states is reduced compared to the situation just after the ionizing light pulse. In previous experiments, recombination processes were inferred from the very low abundance of Xe core ions in Xe-core Ar-shell systems and interpreted in terms of an efficient recombination process within the core [9]. In fluorescence measurements, the signal from clusters was larger than that from atoms, which was explained by recombination processes accompanied by the emission of photons [10].

Theoretical calculations have shown that recombined ions or atoms can be reionized in different ways. For instance, the laser pulse that initially induced the cluster ionization can reionize the atoms or ions that result from electron-ion recombination later during the pulse [4]. Also, as we have shown recently [11], the dc electric field of an ion or electron detector can lead to the reionization of highlying Rydberg atoms formed by recombination processes
[12], leading to the production of very slow electrons. Another possibility is the reionization of excited atoms generated during the cluster expansion by a second laser pulse with a low photon energy. This mechanism, which we term reionization of excited atoms from recombination (REAR), provides a way to obtain state-specific spectroscopic signatures of excited states that are formed by recombination processes, which are not accessible by single-pulse experiments.

In this Letter, we present the first time-resolved study of recombination processes occurring in highly ionized raregas clusters, where the ionization is initiated by intense XUV pulses from a high-order harmonic generation (HHG) source. During the nanoplasma expansion, initially quasifree electrons occupy atomic excited states. REAR is achieved by a near-infrared (NIR) or visible (VIS) light pulse and manifests itself by the appearance of specific peaks in measured photoelectron spectra. In Ar clusters, strong contributions resulting from the population of atomic excited states are identified. The electron and ion yields due to REAR increase for pump-probe time delays up to 10 ps and remain constant for longer time delays, up to $5 \mathrm{~ns}$. We observe as well a time-dependent variation of the ion and photoelectron kinetic energy spectra resulting from reionization of the excited atoms during the cluster expansion, even for large delays between the pump and probe pulses; this is attributed to the decreasing influence of the shallow cluster potential during the expansion. In this way, REAR can be employed to trace cluster dynamics up to the ns range. While the parameters in our setup are different from recent rare-gas cluster experiments using intense $x$-ray pulses from the Linac Coherent Light Source [2,3], qualitatively similar processes are expected to take place in both experimental conditions.

For the experiments, we use a 50-Hz Ti:sapphire laser system with a pulse energy of $35 \mathrm{~mJ}$ and a duration of $32 \mathrm{fs}$ 
[13] to generate high-order harmonics in a 15-cm-long gas cell statically filled with Ar. The HHG beam is filtered by a 200-nm-thick aluminum foil to block the collinearly propagating NIR light, and then focused by a SiC-coated spherical multilayer mirror including an $\mathrm{AlMg}$ protection layer. The mirror has a reflectivity peak at $38 \mathrm{~nm}$ and a focal length of $75 \mathrm{~mm}$.

In the interaction zone, the XUV pulse has a main spectral contribution at $33.1 \mathrm{eV}$ and smaller contributions at 26.8, 23.6, and $20.5 \mathrm{eV}$ with a calculated total energy of $10 \mathrm{~nJ}$. The focused intensity is estimated to be $2 \times 10^{12} \mathrm{~W} / \mathrm{cm}^{2}$, in good agreement with numerical calculations [11]. The XUV beam is crossed by a pulsed cluster beam generated by a piezoelectric valve. For the cluster generation, we use either pure Ar gas or a mixture of 98\% $\mathrm{Ar}$ and $2 \% \mathrm{Xe}$, which is known to assemble itself in a core-shell configuration, where Xe forms the core and $\mathrm{Ar}$ the shell of the cluster $[14,15]$. According to the Hagena scaling law [16], the average cluster size for Ar clusters is $\langle N\rangle=3500$. For Ar-Xe clusters, we estimate the cluster size as $\langle N\rangle=4600$ with $3400 \mathrm{Ar}$ atoms and $1200 \mathrm{Xe}$ atoms, which yields a Xe enrichment factor of 13 in good agreement with [15]. We estimate a moderate cluster density in the interaction zone of around $10^{10} / \mathrm{cm}^{3}$; therefore, multicluster interactions as previously observed in [17] are not expected to take place. A velocity map imaging spectrometer is used to record 2D momentum distributions of the generated ions and electrons, from which we obtain all the presented angle-integrated kinetic energy spectra by an Abel inversion method [18]. More details of the experimental setup are also found in [11].

Prior to the harmonic generation, a beam splitter is used to couple out $10 \%$ of the NIR energy, allowing the configuration of a probe arm with a variable delay. This beam is recombined with the harmonic radiation by a mirror with a 6-mm central hole that reflects the outer part of the probe beam while the harmonic beam propagates through the hole. From there, both beams are sent collinearly towards the focusing mirror. The intensity of the probe beam in the interaction region was calibrated by measuring the $\mathrm{Ar}^{2+} / \mathrm{Ar}^{+}$ratio for atomic samples and by comparison to previously published experimental and theoretical results $[19,20]$. In order to avoid any significant ionization from atoms or clusters by the NIR pulse alone, the peak intensity of the near-infrared laser pulse was subsequently kept below $2 \times 10^{13} \mathrm{~W} / \mathrm{cm}^{2}$. In addition, the second harmonic of the probe beam could be generated by inserting a beta barium borate crystal. Upon the removal of the aluminum foil from the harmonic path, it is possible to perform pump-probe experiments with 790-nm and 395$\mathrm{nm}$ laser wavelengths, where each of the beams can be used as a pump or probe beam.

In the experiment, 2D $\mathrm{Ar}^{+}$ion momentum maps resulting from the ionization of argon clusters by two-color XUV + NIR pulses $(\Delta t=200 \mathrm{ps})$ and by a one-color
XUV pulse were recorded. The difference between the two momentum distributions is presented in Fig. 1(a). Angle-integrated kinetic energy spectra obtained for $\mathrm{XUV}+\mathrm{NIR}$ and for XUV-only excitation are shown as well (along with the difference between them) in Fig. 1(b). It is evident that the NIR laser leads to a significant increase in the number of detected ions. We note that this enhanced ion yield is only visible for clusters and is absent when an atomic target is used. In Fig. 1(c), the evolution of the $\mathrm{Ar}^{+}$ ion kinetic energy distribution is shown as a function of the time delay between the pump and probe pulses. Note that both axes are plotted logarithmically in order to display the XUV background-subtracted kinetic energy spectra between 60 fs and 200 ps, which include low-energy and high-energy distributions. The large ion yield enhancement for time delays up to 8 ps with a broad kinetic energy
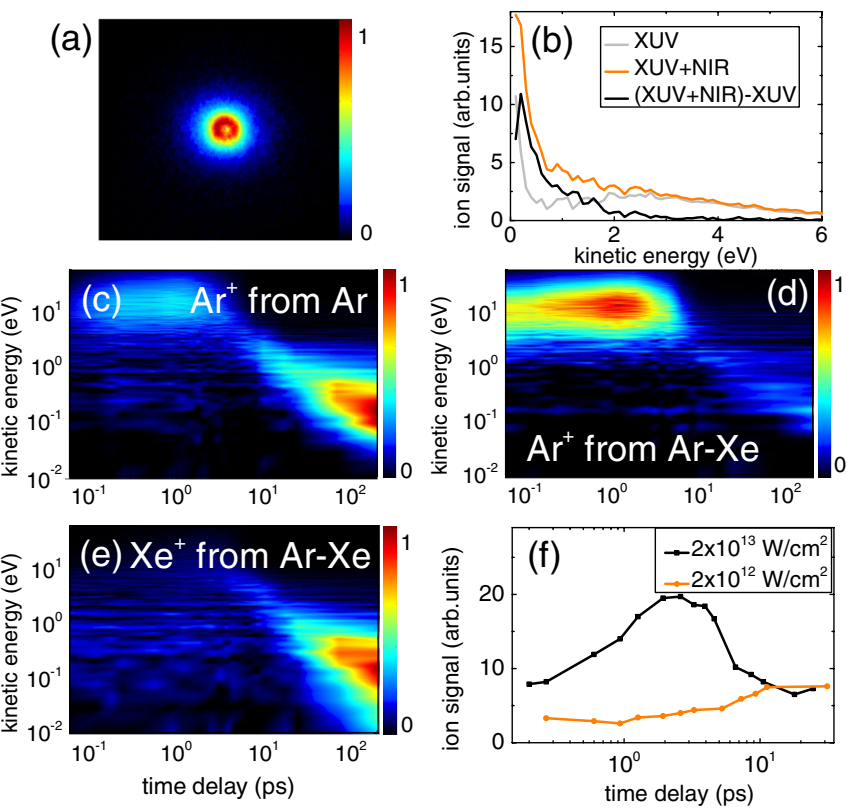

FIG. 1 (color online). (a) Normalized differential 2D momentum distribution of $\mathrm{Ar}^{+}$ions from pure Ar clusters with $\langle N\rangle=$ 3500 ionized with a $38-\mathrm{nm}$ pulse and probed with a $790-\mathrm{nm}$ pulse delayed by 200 ps. The signal obtained with the XUV pulse only was subtracted from the two-color signal. See text for further experimental details. (b) Angle-integrated kinetic energy spectra showing the XUV-only, XUV + NIR and NIR-induced signal. The NIR-induced $\mathrm{Ar}^{+}$ions are observed at energies below $3 \mathrm{eV}$. (c) Time-resolved $\mathrm{Ar}^{+}$kinetic energy spectra as a function of the delay between the XUV pump and the NIR probe pulse in the case of pure Ar clusters, showing a high-energy contribution at time delays $<10 \mathrm{ps}$ and a low-energy contribution for delays $>10 \mathrm{ps}$. As before, signals corresponding to XUVonly $\mathrm{Ar}^{+}$ion production were subtracted. In the case of mixed Xe-Ar clusters with $\langle N\rangle=4600$, the high-energy distribution is mainly observed for $\mathrm{Ar}^{+}$ions from the cluster shell (d), while the low-energy contribution is present for $\mathrm{Xe}^{+}$ ions from the core (e). (f) Time-dependent $\mathrm{Xe}^{+}$ion yield generated in Ar-Xe clusters measured at two different NIR intensities of $2 \times 10^{12} \mathrm{~W} / \mathrm{cm}^{2}$ and $2 \times 10^{13} \mathrm{~W} / \mathrm{cm}^{2}$. 
distribution ranging beyond $60 \mathrm{eV}$ is attributed to wellknown resonance effects, where the NIR laser frequency equals the plasmon frequency of the expanding nanoplasma. A similar dependence was previously observed in experiments where both the pump and probe pulses were in the NIR regime [21-23]. In addition to the large enhancement of the ion yield observed during the first few ps of the cluster expansion, an NIR-induced lowenergy signal $\left(E_{k}<3 \mathrm{eV}\right)$ appears for delays larger than 10 ps. We have also observed this low-energy signal when increasing the time delay between the two pulses to $5.2 \mathrm{~ns}$.

To gain insight into the process leading to the increased ion yield at large time delays, core-shell clusters were employed, where Xe forms the core and Ar the shell. By observing the dynamics of the $\mathrm{Ar}^{+}$and $\mathrm{Xe}^{+}$ions separately [see Figs. 1(d)-1(e)], it is evident that the two-color signal for time delays larger than $10 \mathrm{ps}$ is prominently enhanced for the $\mathrm{Xe}^{+}$ions, and nearly absent for the $\mathrm{Ar}^{+}$ions. The origin of the additional ion signal from the cluster core supports a close connection to the cluster recombination dynamics. During the cluster expansion, recombination of quasifree electrons with ions is more efficient in the cluster core than in the cluster shell, due to the high density of charged particles that is maintained within the slowly expanding core [24]. In this way, low-lying excited states are populated, which can be ionized by the NIR pulse, as well as high-lying Rydberg states that can field ionize in the detector electric field, as observed previously [11].

In order to investigate the time dependence of the recombination process leading to excited state levels, the probe intensity was lowered from $2 \times 10^{13} \mathrm{~W} / \mathrm{cm}^{2}$ to $2 \times 10^{12} \mathrm{~W} / \mathrm{cm}^{2}$. This significantly reduces the electron heating at the plasma frequency resonance, while singlephoton ionization from excited states is still expected to be saturated. We focus on $\mathrm{Xe}^{+}$ions from mixed $\mathrm{Xe}-\mathrm{Ar}$ clusters, since here a large number of recombination processes are observed. As seen in Fig. 1(f), the temporal evolution of the ion signal is different for the two NIR laser intensities. In particular, at the lower intensity, a monotonic increase of the $\mathrm{Xe}^{+}$ion yield is observed up to a time delay of 10 ps. Subsequently the ion yield becomes constant. We take this as a clear indication that excited states are occupied in the first $10 \mathrm{ps}$ after cluster ionization. In contrast, the ion yield exhibits a maximum at a time delay of 3 ps when an intensity of $2 \times 10^{13} \mathrm{~W} / \mathrm{cm}^{2}$ is used.

Further evidence for the population of excited states by electron-ion recombination is obtained by measuring photoelectron spectra resulting from the two-color XUV+NIR experiment. In Fig. 2(a), we present a 2D electron momentum map recorded from Ar clusters for a time delay of $5.2 \mathrm{~ns}$ between the $38-\mathrm{nm}$ pump and the $790-\mathrm{nm}$ probe pulses. Similar to the measurements in Fig. 1, the momentum distribution presented here reflects the difference between an image recorded with both the XUV and NIR pulses present and an XUV-only image. The momentum
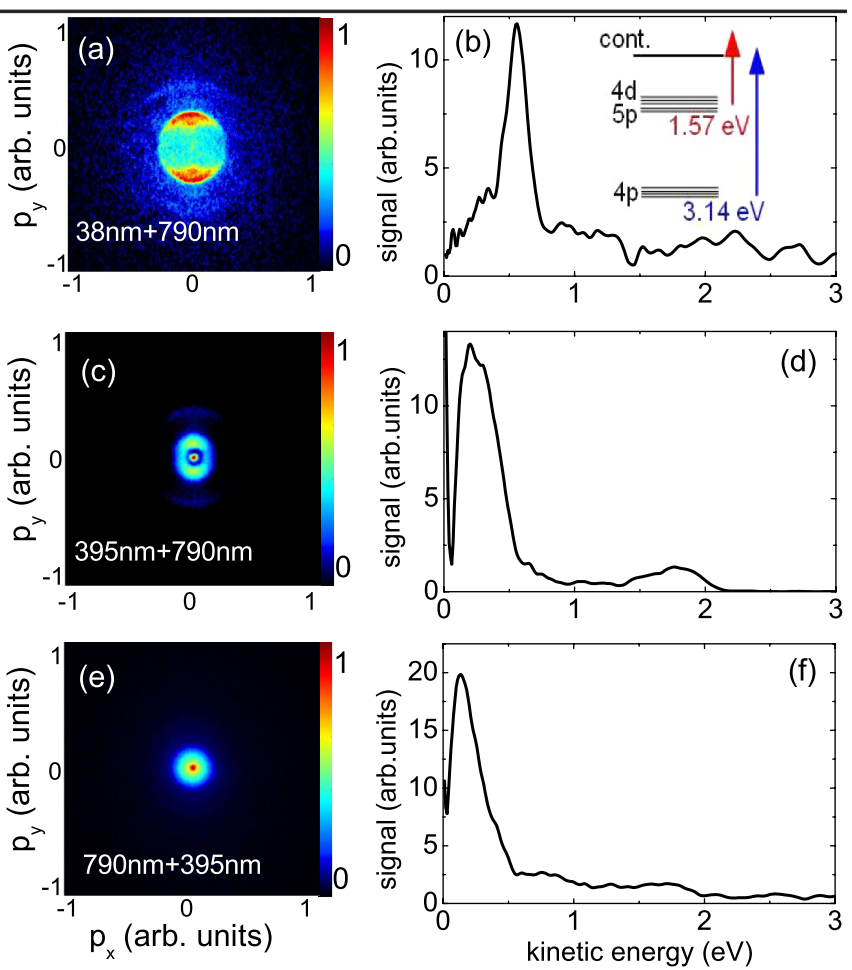

FIG. 2 (color online). (a) Differential two-color electron momentum maps from Ar clusters using a 38-nm pump and a 790-nm probe pulse delayed by 5.2 ns. (b) Angle-integrated kinetic energy spectrum with a clear peak attributed to the reionization of excited atoms. The inset shows a schematic with examples of possible one-photon reionization processes with $1.57 \mathrm{eV}(790 \mathrm{~nm})$ and $3.14 \mathrm{eV}(395 \mathrm{~nm})$ photons from the $5 p$, $4 d$, and $4 p$ states. (c) and (d): Same, after excitation at $395 \mathrm{~nm}$ and probing at $790 \mathrm{~nm}$ with a time delay of $130 \mathrm{ps}$. (e) and (f): Same, pumping at $790 \mathrm{~nm}$ and probing with a 200-ps-delayed 395-nm pulse.

map shows a clear ring structure that is attributed to singlephoton ionization of excited atoms formed during the cluster expansion. In the angle-integrated photoelectron spectrum displayed in Fig. 2(b), a strong peak is visible at an energy of $0.6 \mathrm{eV}$. While an unambiguous assignment to a certain state is not possible, the reionization from several $5 p$ and $4 d$ states of Ar is expected to lead to an electron emission with kinetic energies around $0.6 \mathrm{eV}$. The observed peak structure is a clear indication that the observed increase of the ion yield originates from a REAR process. We note that signal at higher electron energies suggests additional ionization from other excited states.

In addition to recombination, other mechanisms may also lead to the generation of excited atoms. While direct excitation by the XUV pump laser is ruled out due to the large photon energies well above the atomic ionization potential, electron impact excitation can contribute to the observed signal. However, repeating the experiment shown in Figs. 2(a)-2(b) with lower photon energies between 17.3 and $23.5 \mathrm{eV}$ leads to a strong excited atom formation as well. In this case, photoelectrons do not have sufficient 
energy for electron impact excitation from the ground state of Ar. A small fraction of the electrons may gain additional energy, but this cannot explain the strong two-color XUV + NIR signal. While moderate kinetic energies up to $0.85 \mathrm{eV}$ were reported for excited atoms from clusters [25], the larger kinetic energies up to $3 \mathrm{eV}$ observed in Fig. 1(b) suggest that the excited atoms are in a transient ionic state during cluster dissociation. Therefore, we regard recombination as the main process for the formation of excited atoms.

The generation of excited state atoms via recombination is a consequence of nanoplasma formation, which was previously observed over a wide range of laser wavelengths, including the XUV [26] and NIR [27] regimes. Hence, we would also expect the REAR process to be important under these conditions. To demonstrate the universal nature of the REAR process, we have explored the formation of excited states for two different excitation scenarios, where 395-nm and 790-nm pulses, respectively, were used to initiate the nanoplasma generation by strong field ionization. The momentum map in Fig. 2(c) was taken at a time delay of $130 \mathrm{ps}$ between the $395-\mathrm{nm}$ pump and 790-nm probe pulses, and also exhibits a well-defined ring structure attributed to REAR, though at slightly smaller radii than in Fig. 2(a). The angle-integrated spectrum in Fig. 2(d) shows that the main peak is broader and shifted to smaller energies compared to the spectrum in Fig. 2(b). This observation may be due to the population of different states under the changed pump conditions. In addition, a stronger cluster potential at this time delay may influence the kinetic energies of the outgoing photoelectrons (see below). When using a 790-nm pulse as the pump and a 200-ps-delayed 395-nm pulse as the probe beam, the single-photon ionization of lower excited state atoms by the probe beam becomes possible. For instance, the reionization from the $\operatorname{Ar}(4 p)$ state at a wavelength of $395 \mathrm{~nm}$ is expected to result in the emission of electrons with kinetic energies between 0.29 and $0.86 \mathrm{eV}$, which may partially explain the measured electron spectrum in Figs. 2(e) and 2(f).

For time delays larger than $10 \mathrm{ps,} \mathrm{two-color} \mathrm{electron}$ spectra provide a sensitive probe for the cluster expansion. Analogous to the cluster ionization process, where photoelectrons are decelerated by the increasing Coulomb potential [28], probing of excited states formed from the nanoplasma by an NIR field reflects the cluster environment. In Fig. 3, differential two-color electron spectra are presented for different time delays between the XUV pump and NIR probe pulses. In the momentum map in Fig. 3(a) taken at a time delay of $67 \mathrm{ps}$, the ring structure present in Fig. 2(a) is hardly discernible. The angle-integrated kinetic energy spectra in Fig. 3(b) show that the dominant peak clearly gets narrower and is shifted to higher energies upon the increase of the time delay, a process that continues up to $5.2 \mathrm{~ns}$. The time evolution of the shape and position of the
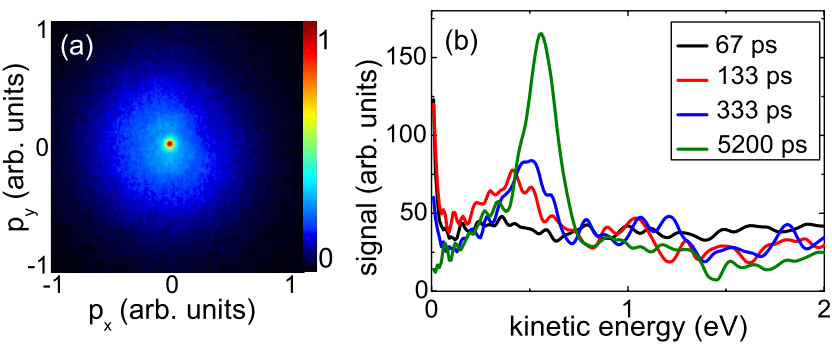

FIG. 3 (color online). (a) Differential two-color electron momentum map for XUV pump and NIR probe pulses with a relative delay of 67 ps. (b) Normalized angle-integrated kinetic energy spectra at different delays of 67 ps, 133 ps, 333 ps, and $5.2 \mathrm{~ns}$. The signal at larger kinetic energies is attributed to the singlephoton ionization from a number of different states as well as to multiphoton ionization processes from these states.

REAR peaks therefore reflects the time evolution of the cluster potential, and possibly also the interaction of the REAR electrons with neighboring ions from previous REAR events. In agreement with the trends observed in Fig. 3(b), these effects induce a kinetic energy downshift that diminishes for very long delays, where the influence of the environment can be neglected and where the REAR process generates sharp photolines from excited atoms in the electron spectra.

In summary, our results show clear evidence for recombination processes to excited atomic states during the expansion of nanoplasmas that result either from cluster ionization by a strong XUV, NIR, or VIS laser pulse. After cluster ionization at different wavelengths, recombined electrons can be reionized by weak laser fields, which in our experiments leads to a large increase of the total ion signal by up to a factor of 2 . We find that in the case of medium-sized Ar-Xe clusters, recombination takes place within 10 ps. In addition, electrons generated via REAR can be exploited to monitor the cluster expansion up to the ns range. Recombination into excited states is expected to take place not only in clusters, but to be a general feature accompanying the interaction of finite systems with intense laser fields. Finally, this first pump-probe investigation of XUV multiphoton processes in clusters with an HHG source paves the way for experiments with attosecond time resolution in the near future, potentially leading to a direct observation of the charging of clusters during ionization with intense XUV pulses.

schuette@mbi-berlin.de

[1] H. Wabnitz et al., Nature (London) 420, 482 (2002).

[2] H. Thomas et al., Phys. Rev. Lett. 108, 133401 (2012).

[3] T. Gorkhover et al., Phys. Rev. Lett. 108, 245005 (2012).

[4] C. Jungreuthmayer, L. Ramunno, J. Zanghellini, and T. Brabec, J. Phys. B 38, 3029 (2005).

[5] U. Saalmann, C. Siedschlag, and J. Rost, J. Phys. B 39, R39 (2006). 
[6] M. Arbeiter and T. Fennel, New J. Phys. 13, 053022 (2011).

[7] E. Ackad, N. Bigaouette, S. Mack, K. Popov, and L. Ramunno, New J. Phys. 15, 053047 (2013).

[8] M. Arbeiter, C. Peltz, and T. Fennel, Phys. Rev. A 89, 043428 (2014).

[9] M. Hoener, C. Bostedt, H. Thomas, L. Landt, E. Eremina, H. Wabnitz, T. Laarmann, R. Treusch, A. R. B. de Castro, and T. Möller, J. Phys. B 41, 181001 (2008).

[10] H. Iwayama, M. Nagasono, J. R. Harries, and E. Shigemasa, Opt. Express 20, 23174 (2012).

[11] B. Schütte, M. Arbeiter, T. Fennel, M. J. J. Vrakking, and A. Rouzee, Phys. Rev. Lett. 112, 073003 (2014).

[12] T. Fennel, L. Ramunno, and T. Brabec, Phys. Rev. Lett. 99, 233401 (2007).

[13] G. Gademann, F. Ple, P.-M. Paul, and M. J. J. Vrakking, Opt. Express 19, 24922 (2011).

[14] M. Tchaplyguine, M. Lundwall, M. Gisselbrecht, G. Ohrwall, R. Feifel, S. Sorensen, S. Svensson, N. Martensson, and O. Bjorneholm, Phys. Rev. A 69, 031201(R) (2004).

[15] O. G. Danylchenko, Yu. S. Doronin, S. I. Kovalenko, and V. N. Samovarov, Pis'ma v Zhurnal Éksperimental'noř i Teoreticheskoĭ Fiziki 84, 385 (2006) [JETP Lett. 84, 324 (2006)].

[16] O. F. Hagena, Surf. Sci. 106, 101 (1981).
[17] T. M. Trivikram, R. Rajeev, K. P. M. Rishad, J. Jha, and M. Krishnamurthy, Phys. Rev. Lett. 111, 143401 (2013).

[18] M. Vrakking, Rev. Sci. Instrum. 72, 4084 (2001).

[19] C. Guo, M. Li, J. P. Nibarger, and G. N. Gibson, Phys. Rev. A 58, R4271 (1998).

[20] A. Becker and F. Faisal, J. Phys. B 32, L335 (1999).

[21] J. Zweiback, T. Ditmire, and M. D. Perry, Phys. Rev. A 59, R3166 (1999).

[22] S. Zamith, T. Martchenko, Y. Ni, S. A. Aseyev, H. G. Muller, and M. J. J. Vrakking, Phys. Rev. A 70, 011201 (R) (2004).

[23] J. Passig, R. Irsig, N.X. Truong, T. Fennel, J. Tiggesbäumker, and K. H. Meiwes-Broer, New J. Phys. 14, 085020 (2012).

[24] C. Peltz and T. Fennel, Eur. Phys. J. D 63, 281 (2011).

[25] O. Bünermann, O. Kornilov, D. J. Haxton, S. R. Leone, D. M. Neumark, and O. Gessner, J. Chem. Phys. 137, 214302 (2012).

[26] T. Laarmann, M. Rusek, H. Wabnitz, J. Schulz, A. R. B. de Castro, P. Gürtler, W. Laasch, and T. Möller, Phys. Rev. Lett. 95, 063402 (2005).

[27] Y. L. Shao, T. Ditmire, J. W. G. Tisch, E. Springate, J. P. Marangos, and M. H. R. Hutchinson, Phys. Rev. Lett. 77, 3343 (1996).

[28] C. Bostedt et al., Phys. Rev. Lett. 100, 133401 (2008). 